\title{
Review of Literature on Teaching and Learning Geometry and Measurement: A Case of Ghanaian Standards Based Mathematics Curriculum
}

\author{
Samuel Baah-Duodu ${ }^{1}$, Vivian Osei-Buabeng ${ }^{1}$, Ennin Francis Cornelius ${ }^{1}$ \& John Ekow Hegan ${ }^{2}$ \\ ${ }^{1}$ Department of Mathematics/ICT, Agogo Presbyterian Women's College of Education Agogo, A/A Ghana
}

${ }^{2}$ Department of Mathematics/ICT Methodist College of Education Akyem Oda, Ghana

\begin{abstract}
This study aimed at providing a theoretical background for the development of the Geometry and Measurement Strands in the New Ghanaian Standards-Based Curriculum. As such it should be of aid to inform the supervisory committee of the Ghana Education Service, National Council for Curriculum and Assessment (NaCCA), National Teacher Education Curriculum Framework (NTECF) and Ministry of Education, considering the Mathematics section of the current Ghana Curriculum Review project. This report concentrated on progressions in the two strands both within the area of higher-level thinking (what we will refer to as 'pedagogy') and within the area of content ('knowledge'). While much has been written on the theoretical progressions, concerns showed that there is little to be found on progressions that could be of direct assistance to the facilitator (classroom teacher). The main emphasis of the theoretical writing on progressions in geometry tends to be on the increasing sophistication of overall 'understanding' of geometry (how do learners do geometry?); in contrast to the curriculum descriptions of geometry, it tends to focus much more on the content of knowledgelability (what do learners do in geometry?). Findings also revealed that the 'Count Me into Measurement program' is the Learning Framework in Measurement, which aims to describe the stages learners progress through in developing an understanding of measurement. The Learning Framework describes three key stages: Identification of the attribute (direct comparison/partitioning/conservation); Informal measurement (counting units/relating number of units to quantity/comparison of measurements); and Unit structure (replicating a single unit/relating size of units to the number required). Learners are perceived as passing through the same three stages in their understanding of each of length, area, volume/capacity and mass, though not at the same time, as increasing the number of dimensions measured leads to the increasing complexity of the concept.
\end{abstract}

Key Words: Standards Based, Curriculum, Measurement, Geometry.

\section{INTRODUCTION}

In the year 2019, the National Council for Curriculum and Assessment $(\mathrm{NaCCA})$ in collaboration with the Ministry of Education rolled out the new standards-based curriculum for Ghanaian basic schools, which is a demonstration of placing learning at the heart of every classroom and ensuring that every learner receives quality education [1]. Provision of accessible quality education for all is non-negotiable if we are to meet the human capital needs of our country, required for accelerated sustainable national development. It is for this reason that the new curriculum sets out clearly the learning areas that need to be taught, how they should be taught and how they should be assessed. It provides a set of core competencies and standards that learners are to know, understand and demonstrate as they progress through the curriculum from one content standard to the other and from one phase to the next. The curriculum and its related teachers' manual promote the use of inclusive and gender responsive pedagogy within the context of learning-centred teaching methods so that every learner can participate in every learning process and enjoy learning. The curriculum encourages the use of Information and Communication Technologies (ICTs) for teaching and learning - ICTs as teaching and learning materials. 
The new curriculum has at its heart the acquisition of skills in the 4Rs of Reading, wRiting, aRithmetic and cReativity by all learners. It is expected that at any point of exit from a formal education, all learners should be equipped with these foundational skills for life, which are also prerequisites for Ghana becoming a learning nation. The graduates from the school system should become functional citizens in the 4Rs and lifelong learners. They should be digital literates, critical thinkers and problem solvers. The education they receive through the study of the learning areas in the curriculum should enable them to collaborate and communicate well with others and be innovative. The graduates from Ghana's schools should be leaders with a high sense of national and global identity. The curriculum therefore provides a good opportunity in its design to develop individuals with the right skills and attitudes to lead the transformation of Ghana into an industrialised learning nation.

For this reason, the Ministry of Education expects that learners, as a result of the new knowledge, skills and values they have acquired through the new curriculum, will show a new sense of identity as creative, honest and responsible citizens. These are the core values that underpin the identification and selection of the learning areas for this curriculum. These core values serve as fundamental building blocks for developing into learners the spirit of teamwork, respect, resilience and the commitment to achieving excellence. The Ministry endorses a quality learning experience as an entitlement for each of Ghana's school-going girl and boy; the curriculum has rightly focused on learning and learning progression. The Ministry has also endorsed accountability as a critical domain for effective workings of standards-based curriculum.

More importantly the role of the teacher is to make this curriculum work for the intended purpose - to inculcate in learners the core competencies and values and to make learning happen; improve learning outcomes - and the support that teachers need is duly recognised and endorsed. The Ministry of Education supports and continue to support the implementation of the curriculum to include capacity development of all teachers in the new curriculum because teachers matter in the development and delivery of the standardsbased curriculum.

Mathematics forms an integral part of our everyday lives. It is a universal truth that development is hinged on Mathematics. It is the backbone of social, economic, political and physical development of a country. It is a never-ending creative process which serves to promote discovery and understanding. It consists of a body of knowledge which attempts to explain and interpret phenomena and experiences. Mathematics has changed our lives, and is vital to Ghana's future development.

To provide quality Mathematics education, teachers must facilitate learning in the Mathematics classroom. This will provide the foundations for discovering and understanding the world around us and lay the grounds for Mathematics and Mathematics related studies at higher levels of education. Learners should be encouraged to understand how Mathematics can be used to explain what is occurring, predict how things will behave and analyse causes and origins of things in our environment. The Mathematics curriculum has considered the desired outcomes of education for learners at the basic level. Mathematics is also concerned with the development of attitudes and is important for all citizens to be mathematically and technologically literate for sustainable development. Mathematics therefore ought to be taught using hands-on and minds-on approaches which learners will find as fun and adopt as a culture.

Ghana believes that an effective Mathematics education needed for sustainable development should be inquiry-based (NaCCA, Ministry of Education 2019) [2]. Thus Mathematics education must provide learners with opportunities to expand, change, enhance and modify the ways in which they view the world. It should be pivoted on learner-centred Mathematics teaching and learning approaches that engage learners physically and cognitively in the knowledge-acquiring process in a rich and rigorous inquiry-driven environment.

Mathematics learning is an active contextualised process of constructing knowledge based on learners' experiences rather than acquiring it. Learners are information constructors who operate as researchers. 
Teachers serve as facilitators by providing the enabling environment that promotes the construction of learners' own knowledge based on their previous experiences. This makes learning more relevant to learners and leads to the development of critical thinkers and problem solvers. The curriculum is aimed at developing individuals to become mathematically literate, good problem solvers who are capable to think creatively and have both the confidence and competence to participate fully in the Ghanaian society as responsible local and global citizens. The core competencies for Mathematics describe a body of skills that teachers in Mathematics at all levels should seek to develop in their learners. They are ways in which teachers and learners in Mathematics engage with the subject matter as they learn the subject. The competencies presented here describe a connected body of core skills that are acquired throughout the processes of teaching and learning.

Geometry is one of the longest established branches of mathematics. It has an extensive range of applications and repository of historical and cultural background. Geometry has been accorded a central place in mathematical education in Western culture for a considerable period of time. One of the major achievements of classical geometry was the systematic collection by Euclid of the geometrical knowledge of the ancient Greeks. This has, until comparatively recently, formed the basis for much of the geometry taught in schools.

During a period of educational reforms in mathematics in the 1950s and 1960s some new syllabuses (sometimes called 'the new maths') were developed where the emphasis was on formal structures which were predominantly algebraic. At the same time, the range of approaches to geometry was broadened from its traditional Euclidean base (which was reduced in depth) to include the use of transformations, vectors, matrices and some topology.

In recent years many countries have been reviewing the aims, content and approach of their geometry curricula. The 1995 study by the International Commission on Mathematics Instruction (ICMI) [Mammana and Villani, 1998] revealed that no clear consensus was emerging about the outcome of these reviews, [3]. The small scale research study into the geometry curricula of a number of countries commissioned in 2000 by the Qualifications and Curriculum Authority (QCA) for England confirmed this.

Against this background this study considered the rationale for a geometry curriculum, its possible content and issues concerned with its effective teaching. Our report sets out a number of recommendations on issues where the working group reached a consensus view. There are some matters on which the working group did not address nor reach a conclusion, and which others may wish to pursue further. In order to help identify major issues raised, the report structured around a number of agreed Key Principles and recommendations. These are presented together with explanations, supporting arguments and, where available, evidence.

Within the standard based curriculum, measurement and geometry are merged together as a strand which is internationally accepted; The National Numeracy Strategy (DfEE, 1998) in the United Kingdom, which is also widely used internationally, and several other countries and regions including Hungary, Italy, Alberta (Canada), and British Columbia (Canada), group the two together in a strand called Space, Shape and Measures or similar [4], [5]. While we are used to considering measurement as a category of its own, grouping it with geometry in this way does resolve several issues with regard to certain topics. For example, when measuring area, clearly the geometric properties of shapes should be brought to bear. Angle, similarly, does not fit fully within either measurement or geometry; when referring to angle as a property of a shape we place it within geometry, but when measuring with a protractor, clearly measurement is more appropriate. Time, money and estimation are also topics that are often included within the scope of the measurement strand, but which could be seen as more logically positioned within the number strand 
(estimation is currently placed within number in the Ghanaian curriculum) as their use is largely focussed around number rather than measurement.

The strand Geometry and Measurement in the Ghanaian standards based curriculum consists of;

- Lines and Shapes

- Position Transformation

- Measurements

- Geometrical Reasoning

For the purposes of this review however, geometry and measurement is treated separately, with the recommendation that consideration be given to ending their status as separate strands if not now in future for curriculum review.

The TIMSS (Trends in Mathematics and Science Study) 2003 framework is intended to describe "important content for learners to have learned in mathematics and science" (Mullis et al., 2003, p. i), [6]. Within the Geometry strand the objectives are grouped into five categories:

- Lines and angles

- Two- and three-dimensional shapes

- Congruence and similarity

- Locations and spatial relationships

- Symmetry and transformations

\section{THEORETICAL AND CULTURAL PERSPECTIVES OF CONCEPT FORMATION}

Mathematics education researchers have addressed the issue of mathematics concept formation from different perspectives according to Skemp's (1987) and Burn's (1992) explanation of concept formation in Mathematics. Skemp (1987) emphasizes the need to provide children with known experiences in the process of mathematical concept formation [7]. Burns (1992) stresses the importance of children's experience in the real world in the learning of mathematical concepts, [8]. He explains further that children attain equilibrium when their understanding is based on reality rather than perception and that there is a continuous interaction between mental conceptual structures and environment at the state of equilibrium. The implication here therefore is that children's previous experiences play a vital role in the successful formation of mathematical concepts. Burns (1992) further suggests three other factors that influence learners' mathematics learning, these being maturity, physical experience and social interaction, and the "process of equilibrium coordinates these three factors" (p.28), [9].

A look at the literature by Skemp and Burns show that even though both of them mentioned the need for previous knowledge in the process of concept formation, the former's explanation of prior knowledge did not make the issue of culture explicit. Concept formation therefore seems to concentrate on factors that are internal to the learner (learner's cognition, maturation etc) with no emphasis on the culture (Piaget, 1953, 1954; Inhelder \& Piaget, 1958), [10-13]. This approach to concept formation seems to influence the Ghanaian system very much. The tendency is that in the process of mathematics concept formation the communicator of the concept may emphasize the innate ability of the learner and neglect social and cultural factors, which equally play vital roles in the process of concept formation (Vygotsky, 1987),[14].

A look at a lesson observation on the teaching of perimeter as reported by Mereku (2004) succinctly describes a typical situation in curriculum delivery in the Ghanaian classroom, [15]. In this lesson Mereku reports of a grade six teacher who introduced the concept of perimeter by reviewing concepts of polygons 
using cut-out shapes such as squares and hexagons. The teacher showed each shape and asked pupils to describe the properties, such as the number of sides, square corners or right angles. The teacher then drew two rectangles on the chalk board and wrote down the formula for perimeter, ' $p=2(1+w)$. Through questioning she guided pupils to identify lengths and widths of the rectangles and then asked them to substitute these into the formula to calculate the perimeter. The teacher worked one or two examples and asked for some volunteers to try some more on the chalkboard. The teacher then wrote five similar exercises from the mathematics textbook on the chalkboard and asked pupils to copy in their notes books to answer them as homework. Considering the lesson presented shows clearly that concept formation in measurement pays very little attention to culture. Meanwhile the standards based curriculum advocates for connecting mathematical concepts with the context of the learners' world so that it presents realistic opportunities for learning.

Mathematics in the Ghanaian society Like other countries in sub-Sahara Africa (see Zaslavsky, 1978), there are numerous mathematical practices in the Ghanaian society, which may support mathematics learning in schools if they are employed in mathematics teaching in the classroom, [16]. It is possible for instance for a learner to be taught both measurement and shapes in the process of designing. In other words, it is rare to find somebody just learning to measure for the sake of it. Learning (in out- of- school context) in the Ghanaian society is mainly contextual in nature. A dressmaker for instance will teach an apprentice how to measure during the process of designing the dress. The dressmaker will not teach the apprentice how to measure at one time and how to apply the measurement skill in designing at another time.

Measuring is yet another mathematical practice in the Ghanaian society. Ghanaian market women make use of empty tins as a unit of measure, especially in the sale of grains, chilli among others. It may be possible for most Ghanaian rice sellers for instance to tell the number of empty margarine tins full of rice which make up one bag of rice. Liquids such as oil for example are usually measured using empty bottles. Thus some of these empty bottles are used as units of measure all over the country. It is very common for market women, for instance, to tell the number of beer bottles of oil in one 4.5- litre gallon. The use of the stretch of arms in the measurement of length, the measurement of time using the sun's position in the day, and the crow of the rooster is also common. Hence measurement is done very much in the Ghanaian society. Designs especially the local designs used in the Ghanaian fabrics such as the "adinkra" are very good examples of some of the regular and irregular shapes that learners experience in school. "Adinkrahene" for instance could be used in the introduction of the concept of concentric circles. By so doing the learner will have the opportunity to learn the concept of concentric circles and also get the opportunity to learn about the Ghanaian culture. The patterns in "Kente", a traditional Ghanaian clothing usually worn by traditional rulers is similar to the patterns in the twil weaving which Charinder (2002) used in her research on culture activity in mathematics. This is an indication that there may be a possibility of mathematizing (i.e. creating mathematics) from the process of making Kente. Apart from the Kente, twil weaving is also carried out in the Ghanaian society, [17]. The architectural design used in building houses in northern Ghana for example shows a lot of geometric shapes. The roof has the shape of a cone while the house is cylindrical in shape. In farms, the mounds in which farmers cultivate yams have the shape of a cone. Also the cultivation of palm trees for example usually follows a pattern. Farmers usually keep constant intervals between the trees. This can be used in the process of teaching concept of series for example. The collection of Adinkra symbols can be used for teaching concepts in geometry such as shapes, congruence and symmetry (i.e. both line and rotational symmetry).

\subsection{Piaget/ Inhelder}


Piaget and Inhelder's (1956) theory describes the development of the ability to represent space. "Representations of space are constructed through the progressive organization of the child's motor and internalized actions, resulting in operational systems" (Clements and Battista, 2003, p. 422), [18]. The order of development is seen to be: topological (connectedness, enclosure, and continuity); projective (rectiliniarity); and Euclidean (angularity, parallelism, and distance). They describe a sequence of stages in the development of children's ability to distinguish between shapes when drawing them.

The stages are:

Stage 0: scribbles (less than 2)

Stage I: Topological - irregular closed curves to represent circles, squares, etc. (2-4 years)

Stage II: Projective - progressive differentiation of Euclidean shapes (4-7 years)

Stage III: Euclidean - ability to draw Euclidean shapes (7-8 years) (Piaget and Inhelder, 1956, pp. 55-57).

Though Ghanaian young children may be able to operate with some Euclidean concepts it seems more likely that topological, projective and Euclidean notions all develop over time and their usage becomes increasingly integrated as they get more experiences in their real situation.

\subsection{Van Hiele levels}

In the 1950s Pierre van Hiele and Dina van Hiele-Geldof developed a series of thought levels that they perceived as describing a progression of increasing sophistication of understanding of geometry, [19]. Initially, five discrete hierarchical levels were described, numbered 0-4; variations on these levels continue to provide the basis for many models used to understand learning in geometry. These levels have more commonly been renumbered as levels 1-5 (Swafford et al., 1997), and many researchers have described the existence of an earlier, pre-recognitive level (Clements and Battista, 2003, p. 429; Clements et al., 1999),[20-22].

\section{THOUGHT LEVELS}

\subsection{Level 0: Pre-recognitive}

At the pre-recognitive level learners cannot reliably distinguish between different classes of figures. For example, while they may be able to distinguish between squares and circles, they may not be able to distinguish between squares and triangles. The Ghanaian teacher who serves a facilitator must ensure and see this stage as an initial stage of the learner in order to guide their inception of geometric concepts.

\subsection{Level 1: Visual}

At the visual level learners recognize figures by their global appearance, rather than by identifying significant features, for example a rectangle would be recognized as a rectangle "because it looks like a door". Some researchers (Clements et al, 1999) believe that this level can be better described as syncretic, as learners at this level often use a combination of verbal declarative and visual knowledge to differentiate between shapes, [23]. That is, at Level 1 some children may apply a combination (synthesis) of overall visual matching with limited feature analysis to identify shapes. Several of these figures and shapes made from materials of different kinds should be explored by the learner. 


\subsection{Level 2: Descriptive/Analytic}

At the descriptive/analytic level learners differentiate between shapes by their properties. For example a learner might think of a rectangle as a shape with four sides, and label all shapes with four sides as rectangles. However they might refuse to accept a square as a rectangle "because it is a square". Here the teacher could employ the use of several examples and counter examples to build up on the learners' convictions on such properties.

\subsection{Level 3: Abstract/Relational}

At the abstract/relational level learners relate figures and their properties. They can provide definitions, and differentiate between necessary and sufficient conditions for a concept. They can classify figures hierarchically, and produce some geometric arguments. Learners could be enhanced with use of the right terminologies in their descriptions, differentiations and definitions.

\subsection{Level 4: Formal deduction}

At the formal deduction level learners develop sequences of statements that logically justify a conclusion; constructing simple, original proofs.

\subsection{Level 5: Rigor}

At the final level, learners rigorously apply rules to derive proofs within a mathematical system. Several problems in real life situations should be employed for learners to apply derived proofs.

\section{PHASES OF LEARNING}

As well as the levels of understanding the van Hieles also described 5 phases of learning through which learners can be taken in advancing to the next level (Hoffer, 1983, p. 208), [24].

\subsection{Phase 1: Inquiry}

In this phase the teacher engages the learner in two-way conversation about the topic. Vocabulary is established and the teacher sets the ground for further study.

\subsection{Phase 2: Directed orientation}

Here the teacher directs the path of exploration in such a way as to ensure that the learner becomes familiar with specific key ideas related to the topic.

\subsection{Phase 3: Expliciting}

Now the learners work much more independently, refining their understanding and use of vocabulary.

Phase 4: Free orientation

In this phase the learners encounter multi-step tasks with no one route to solution, and explore their own methods to obtain solutions.

\subsection{Phase 5: Integration}

Finally the learners review their learning and produce an overview of their understanding. The teacher aids them in summarizing their key ideas. 


\section{Development of geometric proof skills}

Clements and Battista (1992, p. 439) describe three levels of the development of proof skills, [25]:

- Level 1 (Up to age 7-8): At this level there is no integration of ideas.

- Level 2 (7-8 through to 11-12): At this level learners begin to make predictions on the basis of results they have seen in previous experiments. For example, they may, after experimenting with triangles, state that the angles add to make a straight line for each triangle.

- Level 3 (Ages 11-12 and beyond): At this level learners are able to apply deductive reasoning to any assumptions.

\subsection{Spatial representation}

Rosser et al. (1988) describe a sequence of mastery of conceptualization of geometry operations related to reproduction of a simple pattern, [26]. The sequence is:

I. Reproducing a geometric pattern, constructed from blocks.

II. Reproducing a similar pattern, which was covered after an initial 6 second observation period.

IIIA. Reproducing the result after rotation of a similar pattern, which was covered after an initial 6 second observation period, and then rotated.

IIIB. Reproducing a perspective view of a similar pattern, with the original pattern remaining available.

\subsection{Curriculum Implementation of Theories by Teachers}

In Ghana, mathematics teachers begin their career by earning a bachelor's degree by completing a teacher preparation program. Some aspiring math teachers also earn a master's degree in math or education. Course work includes upper-level math classes and teaching methods classes. Before graduating, learners must work in high school classroom under the supervision of a math teacher. Amongst daily tasks which include planning lessons, creating sample problems, and assessing learners, math teachers follow a state or district curriculum and use text books to facilitate and impart knowledge to learners.

Within the geometry and measurement strand, there are content standards and core competencies related to shape and space can be broadly grouped into five categories: describing and classifying shapes; making patterns, shapes and objects; symmetry and transformations; position and movement; and angles. The performance indictors within each of these categories describe a clear progression of complexity from simplest to most complex. For example, the progression of a performance indicator related to direction and movement is:

Reception: Use everyday words to describe position, direction and movement.

Basic 1: Use everyday language to describe position, direction and movement.

Basic 2: Use mathematical vocabulary to describe position, direction and movement.

Basic 3: Read and begin to write the vocabulary related to position, direction and movement.

Basic 4: Recognise positions and directions. Use the eight compass directions.

Basic 5: Recognise positions and directions. Read and plot coordinates in the first quadrant. 
Basic 6: Read and plot coordinates in all four quadrants.

\subsection{National Council of Teachers of Mathematics Standards}

Principles and Standards for School Mathematics (NCTM, 2000) describes objectives for learners from prekindergarten to grade 12, [27]. Within the geometry strand, the objectives are grouped into three categories:

Specify locations and describe spatial relationships using coordinate geometry and other representational systems; Apply transformations and use symmetry to analyze mathematical situations; and Use visualization, spatial reasoning, and geometric modeling to solve problems. The objectives within each of these categories describe a clear progression of complexity from simplest to most complex.

\subsection{Exemplars}

The Ghanaian standards based Curriculum Exemplars refers to support and guidance, which clearly explains the expected outcomes of an indicator and suggests what teaching and learning activities could, take to support the facilitators/teachers in the delivery of the curriculum and describes a progression in development of spatial understanding related to Ghanaian cultures. (NaCCA, 2019; p.19), [28]. Most exemplars must be real life applicable and must include the concepts below;

- Geometric Knowledge

○ Angle

○ Two dimensional and three dimensional shapes

- Geometric Operations

- Angle

○ Symmetry and Transformations

○ Construction and Drawing

\subsection{Count Me into Space}

The Count Me into Shape aims to support teachers by giving a framework for space related mathematics. The framework consists of two key aspects: part-whole relationships, referring to how a shape can be part of a larger shape, and features of shapes; and orientation and motion, referring to perspective and manipulation of shapes. Each of these aspects is seen to have the same sequence of development of types of strategies.

- Emerging strategies: Learners are beginning to attend to spatial experiences, exploring grouping like objects, and assigning words to groups of shapes and concepts related to position and movement.

- Perceptual strategies: Learners are attending to spatial features and beginning to make comparisons, relying particularly on observations and manipulations of physical materials.

- Pictorial imagery strategies: Learners are developing mental images associated with concepts and increasingly using standard language to describe their understanding.

- Pattern and dynamic imagery strategies: Learners are developing conceptual relationships and using pattern and movement in their mental imagery.

- Efficient strategies: Learners select from a range of spatial strategies for a given context. They use imagery, classification, part-whole relationships and orientation. 


\subsection{Measurement}

Lehrer (2003) describes the conceptual development of measurement as "change in a network or web of ideas about measurement". He lists eight components that provide a basis for this network (p. 181), [29]:

1. Unit-attribute relations: What units can/should be used?

2. Iteration: Subdivision into congruent parts or repetition of a unit.

3. Tiling: Gaps must not be left between the units.

4. Identical units: If units are identical a count represents the measure.

5. Standardization: Using a standard unit makes communication of measures easier (possible).

6. Proportionality: Different units can be used to measure and can be compared.

7. Additivity: A line segment can be divided into several smaller line segments whose sum will equal the original length.

8. Origin: When using a scale to measure it is important to identify the zero point, although this can be any point on the scale.

These eight components are seen as important in the development of understanding of all measurement attributes (length/area/volume/mass/angle/time). The development of a conceptual grasp of measures of the different attributes is neither simultaneous nor sequential in a linear way, but understanding of the eight components can be extended from one attribute to another. For example, a learner who understands that when measuring a length using hand spans it is important not to leave gaps, is more likely to understand that measuring an area using tiles must also leave no gaps.

Lehrer et al. (2003) describe a similar set of 'central concepts', with two significant differences: the addition of the concept of precision - that all measurement is inherently approximate and the choice of units (based on the context) determines the level of precision; and the omission of standardization from the list, [30].

\subsection{Curriculum Implementation}

Similarly measurement can be broadly grouped into four categories: vocabulary and units; estimating and measuring; calculating lengths, capacity, mass and time. The content standards within each of these categories describe a clear progression of complexity from simplest to most complex. For example, the progression of objectives related to vocabulary and units is:

Reception: Use language such as more or less, longer or shorter, heavier or lighter...

Basic 1: Understand and use the vocabulary related to length, mass and capacity.

Basic 2: Use and begin to read the vocabulary related to length, mass and capacity.

Basic 3: Read and begin to write the vocabulary related to length, mass and capacity. Begin to use decimal notation for metres and centimetres.

Basic 4: Use, read and write standard metric and imperial units. Know and use the relationships between familiar units of length, mass and capacity. Begin to convert between smaller and larger units.

Basic 5: Use, read and write standard metric units. Convert larger to smaller units. Know imperial units.

Basic 6: Use, read and write standard metric units. Convert larger to smaller units. Know imperial units and their rough equivalents. 
International Journal of Advances in Scientific Research and Engineering (ijasre), Vol 6 (3), March -2020

\section{NATIONAL COUNCIL OF TEACHERS OF MATHEMATICS PRINCIPLES AND STANDARDS}

Principles and Standards for School Mathematics (NCTM, 2000) describes objectives for learners from prekindergarten to grade 12, [31]. Within the measurement strand, the objectives are grouped into two categories:

Understand measurable attributes of objects and the units, systems, and processes of measurement; and

Apply appropriate techniques, tools, and formulas to determine measurements.

The objectives within each of these categories describe a clear progression of complexity from simplest to most complex.

\subsection{Exemplars}

Exemplars include describing a progression in development of understanding of the concept of measurement as applied to length.

Direct comparison

Indirect comparison

Non-standard units

Standard units

\subsection{Use of Reasoned Measurement}

While this sequence of stages is considered to be the same for length, area, volume and mass, the levels are given for length comparisons, as learners are likely to develop this understanding before the more complex attributes of area, volume and mass. Other categories include;

- Time

- Position

- Metric Measurement

\subsection{Count Me into Measurement}

The core of the Count Me into Measurement is the Learning Framework in Measurement, which aims to describe the stages learners' progress through in developing an understanding of measurement. The Learning Framework describes three key stages:

Identification of the attribute (direct comparison/partitioning/conservation); Informal measurement (counting units/relating number of units to quantity/comparison of measurements); and Unit structure (replicating a single unit/relating size of units to number required) (Outhred et al., 2003, p. 85), [32]. Learners are perceived as passing through the same three stages in their understanding of each of length, area, volume/capacity and mass, though not at the same time, as increasing the number of dimensions measured leads to increasing complexity of concept. 


\subsection{Trends in Mathematics and Science Study (TIMSS)}

The TIMSS (Trends in Mathematics and Science Study) 2003 framework is intended to describe "important content for learners to have learned in mathematics and science" (Mullis et al., 2003, p. i), [33]. Within the Measurement strand the objectives are grouped into two categories:

- Attributes and units

- Tools, techniques, and formulas

\subsection{Pedagogical Approaches}

These include the approaches, methods, strategies and appropriate relevant teaching and learning resources for ensuring that every learner benefits from the teaching and learning process. The curriculum emphasizes the:

1. creation of learning-centered classrooms through the use of creative approaches to ensure learner empowerment and independent learning;

2. positioning of inclusion and equity at the center of quality teaching and learning;

3. use of differentiation and scaffolding as teaching and learning strategies for ensuring that no learner is left behind;

4. use of Information Communications Technology (ICT) as a pedagogical tool;

5. identification of subject specific instructional expectations needed for making learning in the subject relevant to learners;

6. integration of assessment as learning, for learning and of learning into the teaching and learning processes and as an accountability strategy; and

7. questioning techniques that promote deep learning.

\subsubsection{Learning-Centered Pedagogy}

The learner is at the center of learning. At the heart of the national curriculum for change and sustainable development is the learning progression and improvement of learning outcomes for Ghana's young people with a focus on the 4Rs - Reading, wRiting, aRithmetic and cReativity. It is expected that at each curriculum phase, learners would be offered the essential learning experiences to progress seamlessly to the next phase. Where there are indications that a learner is not sufficiently ready for the next phase a compensatory provision through differentiation should be provided to ensure that such a learner is ready to progress with his/her cohort. At the primary school, the progression phases are KG1 to KG2 and B1 to B6.

The Curriculum encourages the creation of a learning-centered classroom with the opportunity for learners to engage in meaningful "hands-on" activities that bring home to the learner what they are learning in school and what they know from outside of school. The learning-centered classroom is a place for the learners to discuss ideas through the inspiration of the teacher. The learners, then, become actively engaged in looking for answers, working in groups to solve problems. They also research for information, analyze and evaluate information. The aim of the learning-centered classroom is to enable learners take ownership of their learning. It provides the opportunity for deep and profound learning to take place. The teacher as a facilitator needs to create a learning environment that:

1. makes learners feel safe and accepted;

2. helps learners to interact with varied sources of information in a variety of ways;

3. helps learners to identify a problem suitable for investigation through project work; 
4. connects the problem with the context of the learners' world so that it presents realistic opportunities for learning;

5. organizes the subject matter around the problem, not the subject;

6. gives learners responsibility for defining their learning experience and planning to solve the problem; Importance of play

Play is seen to be important (Owens and Perry, Undated, p. 102; Holton et al., 2001) in the development of understanding of all areas of mathematics, [34], [35], [36]. The importance of explorative investigations in an informal context is seen to have advantages in

(i) the development of understanding of concepts;

(ii) the seeing relations between concepts;

(iii)remembering; and

(iv) bringing enjoyment to the subject.

\section{USE OF INFORMATION AND COMMUNICATION TECHNOLOGY}

Information and Communication Technology (ICT) has been integrated into the Mathematics curriculum as part of the core of education, alongside reading, writing and numeracy. Thus, the curriculum is designed to use ICT as a teaching and learning tool to enhance deep and independent learning. For instance, the teacher, in certain instances, is directed to use multimedia to support the teaching and learning process. ICT has the potential to innovate, accelerate, enrich and deepen skills. It also motivates and engages learners to relate school experiences to work practices. It provides opportunities for learners to fit into the world of work. Some of the expected outcomes that this curriculum aims to achieve are:

1. improved teaching and learning processes; 2 . improved consistency and quality of teaching and learning; 3. increased opportunities for more learner-centered pedagogical approaches; 4. improved inclusive education practices; 5 . improved collaboration, creativity, higher order thinking skills; and 6. enhanced flexibility and differentiated approach of delivery

The use of ICT as a teaching and learning tool is to provide learners an access to large quantities of information online and offline. It also provides the framework for analyzing data to investigate patterns and relationships in statistical data]. Once learners have made their findings, ICT can help them organize, edit and print the information in many different ways.

Many researchers in the late 1980s including (Olson et al, 1987) have written on the value of Logo in helping children to develop an understanding of many important principles of geometry and measurement, [37]. Logo's use has also often been linked to the van Hiele levels, due to the fact that procedures used in Logo can be seen to be developed in a similar way to the van Hiele levels. There are also a number of pieces of software that are currently in use in classrooms, including Geometer's Sketchpad, Excel, and Omnigraph (Perks et al, 2002), all of which have value if used to help develop ideas (through play and experimentation) rather than simply as a calculating tool, [38].

Interactive whiteboards have also been demonstrated to be a valuable tool for teaching mathematics (Ball, 2003), [39]. Because they can be used in conjunction with other computer programs they are particularly valuable when linked to programs such as Geometer's Sketchpad. 
NaCCA, Ministry of Education since 2019 entreats all basic school mathematics tutors to observe several guidelines and strategies which is applicable to all strands including the teaching and learning of Geometry and Measurement, [40].

\subsection{Instructional Expectations}

1. Guide and facilitate learning by generating discourse among learners and challenging them to accept and share responsibility for their own learning, based on their unique individual differences.

2. Select Mathematics content, adapt and plan lessons to meet the interests, knowledge, understanding, abilities, and experiences of learners.

3. Work together as colleagues within and across disciplines and grade levels to develop communities of Mathematics learners who exhibit the skills of mathematical inquiry and the attitudes and social values conducive to mathematics learning.

4. Use multiple methods and systematically gather data about learner understanding and ability to guide Mathematics teaching and learning with arrangements to provide feedback to both learners and parents.

5. Design and manage learning environments that provide learners with the time, space and resources needed for learning Mathematics.

\subsection{Core Competencies}

The core competencies for Mathematics describe a body of skills that teachers in Mathematics at all levels should seek to develop in their learners. They are ways in which teachers and learners in Mathematics engage with the subject matter as they learn the subject. The competencies presented here describe a connected body of core skills that are acquired throughout the processes of teaching and learning.

Critical Thinking and Problem Solving (CP)

This skill develops learners' cognitive and reasoning abilities to enable them analyze and solve problems. Critical thinking and problem solving skill enables learners to draw on their own experiences to analyze situations and choose the most appropriate out of a number of possible solutions. It requires that learners embrace the problem at hand, persevere and take responsibility for their own learning.

Creativity and Innovation (CI)

Creativity and Innovation promotes entrepreneurial skills in learners through their ability to think of new ways of solving problems and developing technologies for addressing the problem at hand. It requires ingenuity of ideas, arts, technology and enterprise. Learners having this skill are also able to think independently and creatively.

Communication and Collaboration (CC)

This competence promotes in learners the skills to make use of languages, symbols and texts to exchange information about themselves and their life experiences. Learners actively participate in sharing their ideas. They engage in dialogue with others by listening to and learning from them. They also respect and value the views of others.

Cultural Identity and Global Citizenship (CG) 
This competence involves developing learners to put country and service foremost through an understanding of what it means to be active citizens. This is done by inculcating in learners a strong sense of social and economic awareness. Learners make use of the knowledge, skills, competencies and attitudes acquired to contribute effectively towards the socio-economic development of the country and on the global stage. Learners build skills to critically identify and analyze cultural and global trends that enable them to contribute to the global community.

\subsection{Personal Development and Leadership (PL)}

This competence involves improving self-awareness and building self-esteem. It also entails identifying and developing talents, fulfilling dreams and aspirations. Learners are able to learn from mistakes and failures of the past. They acquire skills to develop other people to meet their needs. It involves recognizing the importance of values such as honesty and empathy and seeking the well-being of others. Personal development and leadership enable learners to distinguish between right and wrong. The skill helps them to foster perseverance, resilience and self-confidence. PL helps them acquire the skill of leadership, selfregulation and responsibility necessary for lifelong learning.

Digital Literacy (DL)

Digital Literacy develops learners to discover, acquire and communicate through ICT to support their learning. It also makes them use digital media responsible.

\subsection{Learning Domains (Expected Learning Behaviors)}

A central aspect of the standards based curriculum is the concept of three integral learning domains that should be the basis for instruction and assessment. These are: -

Knowledge,

Understanding and Application -

Process Skills -

Attitudes and Values

Knowledge, Understanding and Application

Under this domain, learners may acquire some knowledge through some learning experiences. They may also show understanding of concepts by comparing, summarizing, re-writing etc. in their own words and constructing meaning from instruction. The learner may also apply the knowledge acquired in some new contexts. At a higher level of learning behavior, the learner may be required to analyze an issue or a problem. At a much higher level, the learner may be required to synthesize knowledge by integrating a number of ideas to formulate a plan, solve a problem, compose a story, or a piece of music. Further, learners may be required to evaluate, estimate and interpret a concept. At the last level, which is the highest, learners may be required to create, invent, compose, design and construct. These learning behaviors "knowing ", "understanding", "applying", "analyzing", "synthesizing", "evaluating" and "creating" fall under the domain "Knowledge, Understanding and Application".

In this curriculum, learning indicators are stated with action verbs to show what the learner should know and be able to do. For example, the learner will be able to describe a geometrical figure like rhombus. Being able to "describe" geometrical figure like rhombus after teaching and learning has been completed means 
that the learner has acquired "knowledge". Being able to explain, summarize and give examples etc. means that the learner has understood the concept taught.

Similarly, being able to develop, defend, etc. means that the learner can "apply" the knowledge acquired in some new context. You will note that each of the indicators in the curriculum contains an "action verb" that describes the behavior the learner will be able to demonstrate after teaching and learning has taken place. "Knowledge, Understanding and Application" is a domain that should be the prime focus of teaching and learning in schools. Teaching in most cases has tended to stress knowledge acquisition to the detriment of other higher level behaviors such as applying knowledge.

Each action verb in any indicator outlines the underlying expected outcome. Each indicator must be read carefully to know the learning domain towards which you have to teach. The focus is to move teaching and learning from the didactic acquisition of "knowledge" where there is fact memorization, heavy reliance on formulae, remembering facts without critiquing them or relating them to real world - surface learning - to a new position called - deep learning. Learners are expected to deepen their learning by knowledge application to develop critical thinking skills, explain reasoning, and generate creative ideas to solve real life problems in their school lives and later in their adult lives. This is the position where learning becomes beneficial to the learner.

The explanation and the key words involved in the "Knowledge, Understanding and Application" domain are as follows:

Knowing: This refers to the ability to remember, recall, identify, define, describe, list, name, match, state principles, facts, concepts. Knowledge is the ability to remember or recall material already learned. This constitutes the lowest level of learning.

Understanding: This refers to the ability to explain, summarize, translate, rewrite, paraphrase, give examples, generalize, estimate or predict consequences based upon a trend. Understanding is generally the ability to grasp the meaning of some material that may be verbal, pictorial or symbolic.

Applying: This dimension is also referred to as "Use of Knowledge". It is the ability to use knowledge or apply knowledge, apply rules, methods, principles, theories, etc. to situations that are new and unfamiliar. It also involves the ability to produce, solve, plan, demonstrate, discover etc.

Analysis: This dimension is the ability to break down material/information into its component parts; to differentiate, compare, distinguish, outline, separate, identify significant points etc., ability to recognize unstated assumptions and logical fallacies; and the ability to recognize inferences from facts etc.

Synthesizing: It is the ability to put parts together to form a new whole. It involves the ability to combine, compile, compose, devise, plan, revise, organize, create, generate new ideas and solutions etc.

Evaluating: This refers to the ability to appraise, compare features of different things and make comments or judgment, compare, contrast, criticize, justify, support, discuss, conclude, make recommendations etc. Evaluating refers to the ability to judge the worth or value of some material based on some criteria.

Creating: This is the ability to use information or materials to plan, compose, produce, manufacture or construct other products. From the foregoing, creation is the highest form of thinking and learning, and is therefore the most important behavior. This unfortunately is the area where most learners perform poorly. In order to get learners to develop critical thinking and behavioral skills beginning right from the lower 
primary level, it is advised that you do your best to help your learners to develop analytic and application skills as we have said already.

\section{Skills and Processes}

The mathematical method is the means by which a mathematician solves problems or seeks to gain information about events. Learners should be exposed to situations that challenge them to raise questions and attempt to solve problems. The more often they are faced with these challenges, the more likely they are to develop a positive attitude toward mathematics, and the more likely they are to develop the relevant process skills. Details of each sub-skill in the "Values, Attitudes and Process Skills" dimension are as follows:

Observing: This is the skill of using our senses to gather information about objects or events. This also includes the use of instruments to extend the range of our senses.

Classifying: This is the skill of grouping objects or events based on common characteristics.

Comparing: This is the skill of identifying the similarities and differences between two or more objects, concepts or processes.

Communicating/ : This is the skill of transmitting, receiving and presenting information in concise, clear and accurate forms - verbal, written, pictorial, tabular or Reporting graphical.

Predicting: This is the skill of assessing the likelihood of an outcome based on prior knowledge of how things usually turn out.

Analyzing: This is the skill of identifying the parts of objects, information or processes and the patterns and relationships between these parts.

Generating: This is the skill of exploring all the options, possibilities and alternatives beyond the obvious or preferred one Possibility

Evaluating: This is the skill of assessing the reasonableness, accuracy and quality of information, processes or ideas. It also involves assessing the quality and feasibility of objects.

Designing: This is the skill of visualizing and drawing new objects or gargets from imagination

Measuring: This is the skill of using measuring instruments and equipment for measuring, reading and making observations

Interpreting: This is the skill of evaluating data in terms of its worth: good, bad, reliable, unreliable; making inferences and predictions from written or graphical data; extrapolating and deriving conclusions. Interpretation is also referred to as "Information Handling".

Recording: This is the skill of drawing or making graphical representation boldly and clearly, well labeled and pertinent to the issue at hand.

Generalizing: This is the skill of being able to use the conclusions arrived at in an experiment to what could happen in similar situations.

Designing: This is the skill of developing hypotheses; planning and designing of experiments; persisting in the execution of experimental activities and Experiments modifying experimental activities where necessary in order to reach conclusions. Learners therefore need to acquire positive attitudes, values and psychosocial 
skills that will enable them to participate actively in lessons and take a stand on issues affecting them and others.

\subsection{Assessment}

Assessment is a process of collecting and evaluating information about learners and using the information to make decisions to improve their learning. In the standards based curriculum, it is suggested that assessment is used to promote learning. Its purpose is to identify the strengths and weaknesses of learners to enable teachers to ascertain their learner's response to instruction. Assessment is both formative and summative. Formative assessment is viewed in terms of assessment as learning and assessment for learning. Assessment as learning: Assessment as learning relates to engaging learners to reflect on the expectations of their learning. Information that learners provide the teacher forms the basis for refining teaching-learning strategies. Learners are assisted to play their roles and to take responsibility of their own learning to improve performance. Learners set their own goals and monitor their progress. Assessment for learning: It is an approach used to monitor learner's progress and achievement. This occurs throughout the learning process. The teacher employs assessment for learning to seek and interpret evidence which serves as timely feedback to refine their teaching strategies and improve learners' performance. Learners become actively involved in the learning process and gain confidence in what they are expected to learn. Assessment of learning: This is summative assessment. It describes the level learners have attained in the learning, what they know and can do over a period of time. The emphasis is to evaluate the learner's cumulative progress and achievement.

It must be emphasized that all forms of assessment should be based on the domains of learning.

In developing assessment procedures, teachers must try to select indicators in such a way that they will be able to assess a representative sample from a given strand. Each indicator in the curriculum is considered a criterion to be achieved by the learners. When you develop assessment items or questions that are based on a representative sample of the indicators taught, the assessment is referred to as a "Criterion-Referenced Assessment". In many cases, a teacher cannot assess all the indicators taught in a term or year. The assessment procedure you use i.e. class assessments, homework, projects etc. must be developed in such a way that the various procedures complement one another to provide a representative sample of indicators taught over a period.

\section{Summary, Significance and Recommendation}

This report presents the findings of theoretical review to consider for teaching and learning of geometry and measurement in schools and colleges. The study was initiated following the introduction of the standards based curriculum of international educational comparisons, the 2019 revision of the National Curriculum and at a time of several major policy initiatives in education in Ghana.

The working group considered the rationale for geometry and measurement curriculum, its possible content and issues concerned with its effective teaching based on theories. This report reflects its agreed views on the state of the strand and major issues needing to be addressed to bring about improvements.

In order to help identify major issues raised, the report is structured around a number of agreed Key Principles. In the main body of the report these are presented together with explanations, supporting arguments and, where available, evidence, additional information and exemplification are provided.

Overall, for basic school mathematics, we conclude that the geometrical content of the new National Curriculum, with a few adjustments, forms an appropriate basis for a good geometry and measurement education. In order for this to be achieved, however, considerable changes are needed in the way geometry is taught. It is vital that those working to improve mathematics education ensure that their work contributes significantly to improvements in geometry (as well as mathematics) teaching. Bringing about improvements 
in geometry teaching will require a significant commitment to a substantial Programme of continuing professional development alongside the development of appropriate supporting materials.

Though there may be insufficient opportunities for learners to build on their studies in geometry. Those concerned with curriculum design need to review the structure of qualifications in mathematics to ensure they provide improved opportunities for learners to continue to study geometry. The provision of challenging and interesting geometry should help make mathematics a more attractive subject of study for more learners. This in turn would contribute to overcoming the current shortage of Ghanaians with good mathematical skills.

\subsection{Key Principles}

Key Principle 1: Geometry and measurement should form a significant component of the mathematics curriculum for all learners.

Key Principle 2: Any choice of curriculum should be underpinned by a rationale.

Key Principle 3: The geometry curriculum should maintain breadth, depth and balance, and be consistent with Key Principle 2 and the objectives in Recommendation 3.

Key Principle 4: Geometry should be given a higher status, together with a fair share of the teaching time available for mathematics.

Key Principle 5: Learners' education should have the opportunity to continue further their studies in geometry.

Key Principle 6: The assessment framework for the curriculum should be designed to ensure that the full range of learners' geometrical knowledge, skills and understanding are given credit.

Key Principle 7: The most significant contribution to improvements in geometry teaching will be made by the development of good models of pedagogy, supported by carefully designed activities and resources, which are disseminated effectively and coherently to and by teachers.

Key Principle 8: It is a matter of national importance that as many of our learners as possible fully develop their mathematical potential. Geometry, with its distinctive appeal, should make mathematics attractive to a wider range of learners.

\subsection{Significance}

The study and its results would be of aid to mathematics teachers to ensure quality delivery of geometry and measurement based on theory and adequately inform mentoring universities, supervisory committee of the Ghana Education Service, National Council for Curriculum and Assessment (NaCCA), National Teacher Education Curriculum Framework (NTECF) and Ministry of Education, considering the Mathematics section of the current Ghana Curriculum Review Project for teachers' continuous professional development.

\subsection{Recommendations}

Recommendation 1: We recommend that curriculum and assessment specifications be reviewed to ensure that geometry forms a significant component of the mathematics curriculum for all learners.

Recommendation 2: We recommend that the geometry curriculum be chosen and taught in such a way as to achieve the following objectives: a) to develop spatial awareness, geometrical intuition and the ability to visualize; b) to provide a breadth of geometrical experiences in 2 and 3-dimensions; c) to develop 
knowledge and understanding of and the ability to use geometrical properties and theorems; d) to encourage the development and use of conjecture, deductive reasoning and proof; e) to develop skills of applying geometry through problem solving and modeling in real world contexts; f) to develop useful Information \& Communication Technology (ICT) skills in specifically geometrical contexts; g) to engender a positive attitude to mathematics; and h) to develop an awareness of the historical and cultural heritage of geometry in society, and of the contemporary applications of geometry.

Recommendation 3: We recommend that the mathematics teachers develop the desire to encourage learners to work investigatively, demonstrate creativity and make discoveries in geometrical contexts so that learners develop their powers of spatial thinking, visualization and geometrical reasoning.

Recommendation 4: We recommend that the mathematics teachers develop ways which recognize the important position of theorems and proofs within mathematics and use the study of geometry to encourage the development of logical argument appropriate to the age and attainment of the student.

Recommendation 5: We recommend that the mathematics curriculum should provide ample opportunities for learners to use geometry for practical problem solving through mathematical modeling in both 2- and 3dimensions.

Recommendation 6: We recommend that the relevant government agencies work together, with bodies such as the mathematics professional associations, to provide a coherent framework for supporting the development of teaching and learning in geometry. This will involve:

a) The recognition and development of good practice in geometry teaching through pilot studies and research;

b) the design of programmes of continuing professional development and initial teacher education;

c) The production of supporting materials; and

d) The establishment of mechanisms to provide supporting resources, including ICT.

\section{REFERENCE}

[1] Ball, B., (2003). Teaching and learning mathematics with an interactive whiteboard. Micromath, Spring 2003, pp. 4-6.

[2] Burns, M. (1992). How children learn mathematics. NY: Maths Solutions Publications.

[3] Clements, D. (2003). Teaching and Learning Geometry. In J. Kilpatrick, W. G. Martin, \& D. E. Schifter (Eds.), A research companion to principles and standards for school mathematics (pp. 151-177). Reston, VA: National Council of Teachers of Mathematics.

[4] Clements, D., and Battista, M. (2003). Geometry and spatial reasoning. In D. A. Grouws (Ed.), Handbook of research on mathematics teaching and learning (pp. 420-464). New York: Macmillan.

[5] Clements, D., Swaminathan, S., Hannibal, M., and Sarama, J. (1999). Young childrens' concepts of shape. Journal for Research in Mathematics Education, 30, 192-212

[6] Cherinda, M. (2002). The use of a cultural activity in the teaching and learning of mathematics:

Exploring twill weaving with a weaving board in Mozambican classrooms. Unpublished PhD, University of The Witwatersrand, Johannesburg.

[7] Department for Education and Employment (1998). The National Numeracy Strategy -framework for teaching. London: DfEE.

[8] Department of Education and Training (Undated). Count Me Into Measurement. New South Wales Department of Education and Training. 
International Journal of Advances in Scientific Research and Engineering (ijasre), Vol 6 (3), March -2020

[9] Department of Education and Training (Undated). Count Me Into Space. New South Wales Department of Education and Training.

[10] Hoffer, A. (1983). Van Hiele-Based Research. In R. Lesh, and M. Landau (Eds.), Acquisition of mathematics concepts and processes (pp.205-227). London: Academic Press.

[11] Holton, D., Ahmed, A., Williams, H., and Hill, C., (2001). On the importance of mathematical play. International Journal of Mathematics Education in Science and Technology, 32, 3, pp. 401-415.

[12] Inhelder, B., \& Piaget, J. (1958). The growth of logical thinking from childhood to adolescence London: Routledge \& Kegan Paul.

[13] Lehrer, R. (2003). Developing Understanding of Measurement. In J. Kilpatrick, W. G. Martin, \& D. E. Schifter (Eds.), A research companion to principles and standards for school mathematics (pp. 179192). Reston, VA: National Council of Teachers of Mathematics.

[14] Lehrer, R., Jaslow, L., and Curtis, C. (2003). Developing an understanding of measurement in the elementary grades. In D. H. Clements and G. Bright (Eds.), Learning and teaching measurement (pp. 81-99). Reston, VA: National Council of Teachers of Mathematics.

[15] Mereku, K. (2004). Mathematics curriculum implementation in Ghana. Accra: Danjoe production.

[16] Ministry of Education (2019). Mathematics Curriculum for Ghanaian Basic School. National Council for Curriculum and Assessment (NaCCA)

[17] Mullis, I., Martin, M., Smith, T., Garden, R., Gregory, K., Gonzalez, E., Chrostowski, S., and O'Connor, K., (2003). TIMSS Trends in mathematics and science study: Assessment frameworks and specifications 2003. Boston: International Study Center, Lynch School of Education, Boston College.

[18] Mammana, C. \& Villani, V. (Eds.) (1998), Perspectives on the Teaching of Geometry for the 21st Century. Dordrecht: Kluwer.

[19] National Council of Teachers of Mathematics, (2000). Principles and standards for school mathematics. Reston, VA: Author.

[20] Olson, A., Kieren, T., and Ludwig, S., (1987). Linking Logo, levels and language in mathematics. Educational Studies in Mathematics, 18, pp. 359-370.

[21] Outhred, L., Mitchelmore, M., McPhail, D., and Gould, P. (2003). Count me into measurement: A program for the early elementary school. In D. H. Clements and G. Bright (Eds.), Learning and teaching measurement (pp. 81-99). Reston, VA: National Council of Teachers of Mathematics.

[22] Owens, K. (2003). Count me into space: Implementation over two years with consultancy support. Report for the New South Wales Department of Education and Training. NSW DET Professional Support and Curriculum Directorate.[23]

[23] Owens, K. and Perry, B., (Undated). Mathematics K-10 Literature Review. University of Western Sydney. Available online at

http://www.boardofstudies.nsw.edu.au/manuals/pdf_doc/maths_k10_lit_review.doc

[24] Perks, P., Prestage, S., and Hewitt, D., (2002). Does the software change the maths? Micromath, Spring 2003, pp. 4-6.

[25] Piaget, J. (1953). The origins of intelligence in children. London: Routledge \& Kegan

[25] Paul. Piaget, J. (1954). The child's construction of reality. NY: Basic Books.

[26] Piaget, J., and Inhelder, B., (1956). The child's conception of space. Translated by Langdon, F., and Lunzer, J. London: Routledge and Kegan Paul.

[27] Rosser, R. (1988). Order of acquisition of related geometric competencies in young children. Child Study Journal, 18, 75-90. 
International Journal of Advances in Scientific Research and Engineering (ijasre), Vol 6 (3), March -2020

[28] Skemp, R. R. (1987). The psychology of learning mathematics. Hillsdale, NJ Lawrence Erlbaum Associate Inc. Publishers.

[29] Swafford, J., Jones, G., and Thornton, C. (1997). Increased knowledge in geometry and instructional practice. Journal for Research in Mathematics Education, 28, 467-483.

[30] Vygotsky, L. S. (1987). Thinking and speech (N. Minick, Trans). In R. W. Rieber \& A. S. Carton (Eds.), The collected works of L. S. Vygotsky (Vol. 1, pp. 37-288). NY: Plenum Press.

[31] Zaslavsky, C. (1978). African count number and pattern in African cultures. Chicago: Lawrence Hill Books 